\title{
Characterization of antioxidant tapioca starch/polyaniline composites film prepared using solution casting method
}

\author{
${ }^{1,2}$ Azmi, N.S., ${ }^{1 *}$ Kadir Basha, R., ${ }^{1}$ Othman, S.H. and ${ }^{1}$ Mohammed, M.A.P. \\ ${ }^{1}$ Department of Process and Food Engineering, Faculty of Engineering, Universiti Putra Malaysia, 43400 \\ Serdang, Selangor, Malaysia. \\ ${ }^{2}$ Department of Mechanical Engineering Technology. Faculty of Engineering Technology, Universiti Tun \\ Hussien Onn Malaysia, 86400 Pagoh, Muar, Johor.
}

\author{
Article history: \\ Received: 11 October 2018 \\ Received in revised form: 27 \\ November 2018 \\ Accepted: 6 December 2018 \\ Available Online: 11 \\ December 2018
}

Keywords:

Tapioca starch,

Polyaniline,

Mechanical properties,

Radical scavenging

\section{DOI:}

https://doi.org/10.26656/fr.2017.3(4).144

\begin{abstract}
Recently, the incorporation of polyaniline (PANI) as an additive into the biofilm to produce food packaging material is of particular interest due to the need to improve in the properties of the biocomposite film. The aim of this work is to evaluate the effect of concentration and size of PANI loading into the film on the antioxidant properties of PANI/starch film with the analysis of mechanical and Fourier-transform infrared spectroscopy (FTIR) spectra. Composite films with $0.02 \mathrm{~g}, 0.05 \mathrm{~g}$, and $0.1 \mathrm{~g}$ of PANI loading into the tapioca starch film were prepared with different sizes of PANI. The antioxidant and mechanical properties of tapioca starch/PANI composite film properties were investigated. The composites exhibited an increase in free radical scavenging capacity (a measure of antioxidant activity), with increasing of PANI loading in the film irrespective to the size of PANI. However, the highest free radical scavenging activity showed in sample B with the distribution size of PANI range $100 \mu \mathrm{m} \leq \mathrm{x} \leq 125 \mu \mathrm{m}$. In the mechanical test, with the favorable to have more flexibility film rather than hard film, sample B with $0.05 \mathrm{~g}$ of PANI loading showed more flexible with high elongation at break (EAB) compared to others. Based on the FTIR spectra, the starch/PANI film spectra were similar to the starch/glycerol without PANI. However, one notable weak pick near 1455 $\mathrm{cm}^{-1}$ can be found in sample B and C with respective to the concentration of PANI loaded. The incorporation of PANI greatly affected the antioxidant activity of the film which affected the mechanical properties as well.
\end{abstract}

\section{Introduction}

Over the last few years, substantial research has been conducted to develop and apply biocomposites polymer made from a variety of agricultural commodities industrialization with a conductive polymer. The functions of composites material synthesized are to compensate each other deficiencies. By combining natural biodegradable with a synthetic functional polymer which relatively new and can lead to entirely new directions in the research of advanced functional materials for the future (Saikia et al., 2010). Among the many polymers used to develop biodegradable films, starch has been highlighted by the researchers due to its natural polymer from renewable resources, an abundant and low cost which capable of forming continuous thermoplastic materials (González et al., 2016).

Starch is a polysaccharide produced by higher plants as a function of storing energy (Zareh et al., 2011). It is commercially available in the form from the isolation of either from grains such as potato and tapioca. Chemically, starch contains both linear (amylose) and branched (amylopectin) structures. Starch-based films exhibit some of the appropriate physical characteristics which are isotropic, odourless, non-toxic, biodegradable, tasteless, colorless and constitute a good barrier against oxygen transfer (Yan et al., 2012; Jiménez et al., 2013). Nonetheless, starch-based films have been limited due to the poor mechanical, barrier, and thermal properties. Due to these circumstances, the addition of additives such as plasticizers, antioxidant, antimicrobial or others into the matrix will improve the characteristics of the film (Vieira et al., 2011).

Film characteristics are reliant on the cohesion of the polymeric matrix, which in turn is dependent on the structure of the polymer chains, the film obtainment process and the presence of plasticizers agent itself. Incorporation of plasticizers into the starch-based film is 
necessary to increase the flexibility by reducing the polymer intramolecular forces. The most used of plasticizer is glycerol (Chillo et al., 2008; Müller et al., 2008; Liu et al., 2011; Yan et al., 2012). This is because glycerol is more stable and compatible with hydrophilic bio-polymeric packaging film, starch-based film (Cervera et al., 2004; Chillo et al., 2008).

Recently, conducting polymer such as polyaniline (PANI) has attracted attention as an antioxidant material by having the advantage to its free radical scavenging properties. Antioxidant is intended to prevent the presumed deleterious effects of free radicals in human body, and as prevention to the deterioration of fats and other constituents of the food products. PANI has been shown to be a proficient and superior scavengers of 2,2azino-bis-(3-ethylbenzothiazoline-6-sulfonic acid) (ABTS) and 1,1-diphenyl-2picrylhydrazyl (DPPH) free radicals (Nand et al., 2012). Several convectional food science radical scavenging assays and accelerated storage procedures have been altered to use solid conducting polymer to estimate the effectiveness of different samples and preparations. In the previous study, PANI has been incorporated in several matrices such as polyvinyl alcohol, polyethylene, polystyrene, lowdensity polyethylene, chitosan and starch to produce conducting polymer (Annala and Löfgren, 2006; Yavuz et al., 2009; Saikia et al., 2010; Nand et al., 2011; Zareh et al., 2011; Bhadra et al., 2017). However, it is hard to obtain the flexibility with good antioxidant activity composites film without any usage of commercial petroleum-based material. Hence, in this study by using solution blending methods in mixing the starch and PANI powder is investigated to obtain the flexible and good antioxidant properties.

The aim of this work is to evaluate the effect of concentration and size of PANI loading into the film on the antioxidant activity of PANI/starch film with the analysis of mechanical properties and Fourier transform infrared spectroscopy (FTIR). In the present work, starch/PANI composites film is prepared by using solution casting method.

\section{Materials and methods}

\subsection{Materials}

Aniline (Sigma Aldrich (M) Sdn. Bhd) was purified by vacuum distilled under reduced pressure prior to use (Bhadra et al., 2017). Ammonium persulfate (APS), hydrochloric acid $(\mathrm{HCl})$ and glycerol were purchased from Sigma Aldrich (M) Sdn. Bhd. Tapioca starch (food grade) was obtained from LGC Scientific Sdn. Bhd.

\subsection{Synthesis of PANI}

PANI powders were synthesized chemically according to the procedure Nand et al. (2011) with slight modification. $50 \mathrm{~mL}$ of $0.5 \mathrm{M}$ APS solution was added to $50 \mathrm{~mL}$ containing $1 \mathrm{M}$ aniline with $1 \mathrm{M} \mathrm{HCl}$ and vigorously stirred in the ice bath. The mixture was allowed to react for $2 \mathrm{hrs}$, the PANI was stand in the chiller at $5^{\circ} \mathrm{C}$ for $24 \mathrm{hrs}$. Then, the PANI solution was separated using vacuum filtration technique and washed repeatedly using distilled water and methanol until the supernatant becomes colorless. Then, the supernatant was dried in the oven at $40^{\circ} \mathrm{C}$ for $24 \mathrm{hrs}$. The dried PANI was then ground using dry grinder for 10 mins and sieve using several different sizes ranging from $50 \mu \mathrm{m}$ to 250 $\mu \mathrm{m}$.

\subsection{Preparation of starch/PANI biocomposite film}

Several samples with different compositions were developed using the same protocol for solution casting method as in González et al. (2016) with some modification and the compositions of the composite films are listed in Table 1. The homogenous filmforming solution was prepared by dispersing tapioca starch $(4.0 \mathrm{~g} / 100 \mathrm{~g}$ system $)$ in distilled water $(95 \mathrm{~g} / 100 \mathrm{~g}$ system) and was heated gradually up to $70 \pm 5^{\circ} \mathrm{C}$ with stirring. After achieved gelatinization state, glycerol (1.0 $\mathrm{g} / 100 \mathrm{~g}$ system) added with the PANI and kept for 5 mins. Then, the solution underwent sonication for 5 minutes (Chang et al., 2010) to homogenize the solution.

Table 1. Samples composition of different composites.

\begin{tabular}{ccccc}
\hline Sample & size of PANI powder $(\mathrm{x})$ & PANI $(\mathrm{g})$ & Starch $(\mathrm{w} / \mathrm{v})(\%)$ & Glycerol $(\mathrm{g})$ \\
\hline $\mathrm{S}$ & - & - & 4 & - \\
\hline SG & - & - & 4 & 1 \\
\hline A1 & & 0.02 & & 1 \\
A2 & $50 \mu \mathrm{m} \leq \mathrm{x} \leq 100 \mu \mathrm{m}$ & 0.05 & 4 & \\
A3 & & 0.10 & & 1 \\
\hline B1 & & 0.02 & & \\
B2 & \multirow{2}{*}{$100 \mu \mathrm{m} \leq \mathrm{x} \leq 125 \mu \mathrm{m}$} & 0.05 & 4 & 1 \\
B3 & & 0.10 & & \\
\hline C1 & & 0.02 & & \\
C2 & \multirow{2}{*}{$125 \mu \mathrm{m} \leq \mathrm{x} \leq 250 \mu \mathrm{m}$} & 0.05 & 4 & \\
C3 & & 0.10 & & \\
\hline
\end{tabular}


Then, $40 \mathrm{~mL}$ of the solution was poured into $14 \mathrm{~cm}$ internal diameter Petri dishes resting on a levelled surface for casting and the films were dried at room temperature for 4 days. After completely dried, the film was carefully peeled off. The film was stored in the seal plastic film at $57 \%$ of humidity (Xu et al., 2005).

\subsection{Characterization}

\subsubsection{Mechanical analysis}

The mechanical properties were studied using Universal Testing Machine (Instron Model 5566, United State) with a load cell of $10 \mathrm{kN}$ according to ASTM D882-02 (2002). Film sample strips, using a size of 100 x $15 \mathrm{~mm}$, were cut from each kind of composite films. Before testing, the thickness of the sample was measured using a Digital Vernier Caliper (Model MIT-500-196-20, Japan) at ten arbitrary points of the film. Then, the averages of these values were used to calculate the crosssectional area of each sample. The end of each specimen was mounted using a utility tape to prevent cracking and slippage of films during testing. Force (N) and deformation $(\mathrm{mm})$ were recorded with cross-sectional test speed at $10 \mathrm{~mm} / \mathrm{min}$.

\subsubsection{Free radical scavenging activity}

An adaption of the DPPH assay for use with polymeric samples (Hsu et al., 2008; Hsu et al., 2011) was applied for the assessment of the composites film. The $20\left(1 \times 1 \mathrm{~cm}^{2}\right)$ film pieces were added to $20 \mathrm{~mL}$ of a $55 \mu \mathrm{m}$ methanolic DPPH solution. The samples were left to react in the incubator for $24 \mathrm{hrs}$ and after which the absorbance of the supernatant at $516 \mathrm{~nm}$ was measured using a UV Spectrophotometer machine. A blank DPPH solution without sample was also set up as a control. The amount of $\mathrm{DPPH}^{+}$which reacted over $24 \mathrm{hrs}$ period was then calculated after subtracting away the background loss of $\mathrm{DPPH}^{+}$solution without sample.

\subsubsection{Particle size analysis}

Particle size analysis was analysed by using Mastersizer 2000 (Model APA2000, United Kingdom) laser diffractometer for the analysis of size distribution in different group size of PANI. The measurements were conducted using Hydro MU dispersion units with distilled water as a dispersant.

\subsubsection{FTIR}

FTIR spectra were recorded on Thermonicolet (Model Nicolet 6700, United State) spectrometer and transmission spectra were obtained by forming thin transparent potassium bromide $(\mathrm{KBr})$ pellets (Saikia et al., 2010).

\section{Results and discussion}

\subsection{Antioxidant activity}

The antioxidant activity of a material greatly depends on the capability of the material to donate hydrogen for reducing DPPH and therefore the structural conformation of the materials plays a vital role in its antioxidant activity (Saikia et al., 2010). The free radical scavenging capacity, of the starch/PANI composites, are presented in Figure 1. Advantageously, starch itself to be known to have the ability to scavenge the free radical of DPPH although in a very little activity (Saikia et al., 2010). In this study, tapioca starch shows the antioxidant activity with $5.33 \%$ DPPH scavenging activity.

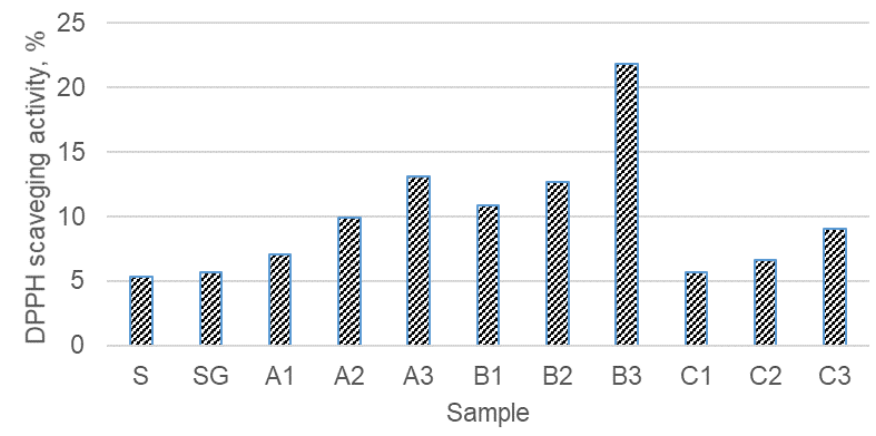

Figure 1. Free radical scavenging activity of different composites film

Many researchers have explored the mechanism of the reaction between aniline and DPPH (Wang et al., 2007; Saikia et al., 2010; Nand et al., 2011; Nand et al., 2012). After $24 \mathrm{hrs}$ reacting PANI with DPPH, the emeraldine salt form gets converted into fully oxidised pernigraniline form which does not possess any hydrogen atom for reducing DPPH. By loading the bulk PANI into the film, it has been observed that the antioxidant property of the composites increases with the increase in the concentration of PANI. This result is in agreement with the findings by Saikia et al. (2010). This trend was repeatedly showed the same for three different size distribution of PANI. This can be attributed to the fact that PANI owing to its redox-active nature is efficient in scavenging the free radical DPPH and such with the increase in the antioxidant activity of the material. This is due to the more hydrogen atom available to be donating to eliminate DPPH. The C-N and $\mathrm{C}_{-} \mathrm{N}^{+}$groups in the PANI structures are important units when it acts as DPPH free radical scavenger. The $\mathrm{C}-\mathrm{N}$ and $\mathrm{C}-\mathrm{N}^{+}$groups are oxidized through $\mathrm{C} \equiv \mathrm{N}$ species is a possible mechanism in this reaction (Hsu et al., 2009).

The result for DPPH radical scavenging capacity of the starch/PANI samples affected by the size of the PANI loaded was in the following order: sample B > sample $\mathrm{A}>$ sample $\mathrm{C}$. The highest DPPH radical scavenging capacity obtained was $21.83 \%$ (sample B3). 
Based on the specific surface area of the particles obtained from the particle size analysis earlier, sample A and sample $\mathrm{B}$ has the slightly similar specific surface area which area $0.127 \mathrm{~m}^{2} / \mathrm{g}$ and $0.1 \mathrm{~m}^{2} / \mathrm{g}$ respectively. Meanwhile, specific surface area sample $\mathrm{C}$ has the smallest specific surface area, $0.0748 \mathrm{~m}^{2} / \mathrm{g}$. It can be noted that sample B was in the lowest oxidation state and thus has the higher capacity to act as reducing agent in scavenging DPPH radicals in compared to sample A and C. Although sample A has the biggest surface area, but the volume of the particle for the mean diameter distribution is lowest than in the sample B. Due to this reason, sample B has the highest scavenging activity. This result is in agreement with Nand et al. (2012), small size of PANI had lower conductivity hence will have the highest radical scavenging activity than the large size of PANI. This further indicates that electroactivity, including the state of oxidation is the critical aspects to be considered of conducting polymer performance in radical scavenging test (Nand et al., 2012).

From Figure 1, it can be seen that the free radical capacity of the composite film increased with the increase in the PANI loading. PANI reduces the DPPH radicals by donating hydrogen atoms, while the PANI segments are in turn oxidized to the pernigraniline form when in contact with DPPH radicals (Nand et al., 2011). Therefore, a higher free radical scavenging capacity was

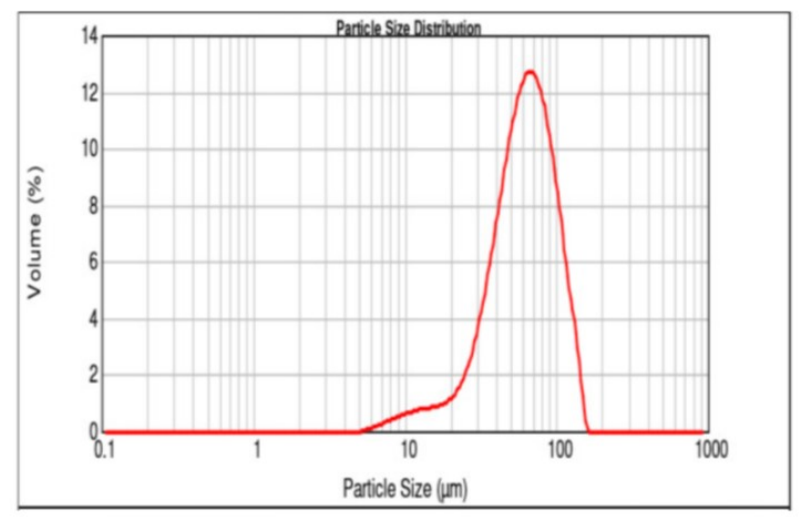

(a) achieved by having a higher concentration of PANI particles being exposed to the DPPH test solution.

\subsection{Mechanical properties}

Table 2. Tensile strength (TS) and Elongation at break (EAB) of the plastic film

\begin{tabular}{ccc}
\hline Sample & TS (Mpa) & EAB $(\%)$ \\
\hline S & 28.89 & 3.28 \\
SG & 8.14 & 12.2 \\
A1 & 7.05 & 8.9 \\
A2 & 7.76 & 3.52 \\
A3 & 9.45 & 3.03 \\
B1 & 5.44 & 11.83 \\
B2 & 4.4 & 14.79 \\
B3 & 3.51 & 11.9 \\
C1 & 6.8 & 6.67 \\
C2 & 5.99 & 10 \\
C3 & 7.6 & 7.3 \\
\hline
\end{tabular}

The mechanical properties of the films are interrelated to the chemical structures of the composites material used (Choi et al., 2017). Tensile strength indicates the maximum stress of films during tensile testing and elongation at break relates to their potential to stretch. The tensile strength (TS) and percentage of elongation at break $(\mathrm{EAB})$ of the different biocomposite film were investigated and plotted as a function of different PANI concentration and size of PANI loaded

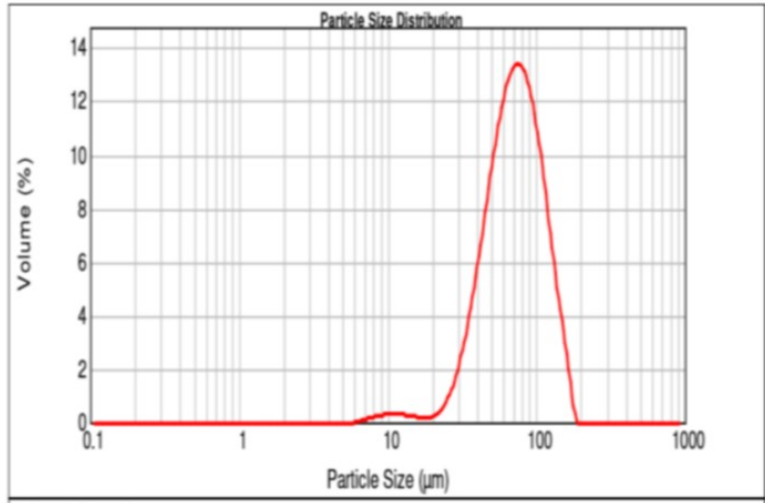

(b)

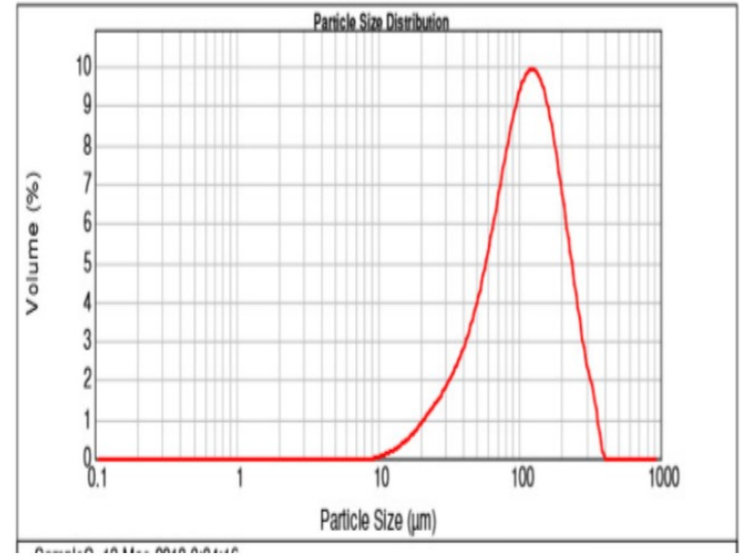

(c)

Figure 2. Particle size distribution for the mesh size of powder PANI (a) $50 \mu \mathrm{m} \leq \mathrm{x} \leq 100 \mu \mathrm{m}$; (b) $100 \mu \mathrm{m} \leq \mathrm{x} \leq 125 \mu \mathrm{m}$; and (c) 125 $\mu \mathrm{m} \leq \mathrm{x} \leq 250 \mu \mathrm{m}$ 
into the film (shown in Table 2). Figure 2 shows the size distribution of the PANI loaded into the film. From Table 2, it is evident that the TS and EAB depended strongly on the concentration and size of PANI loaded into the film.

As in Table 2, the TS of the starch/PANI composite film decreased drastically in compared to starch film without the addition of additives. This relates to the addition of plasticizers or any additive because it is intended to decrease the intramolecular forces along the polymer chains, increasing the film flexibility but decreasing the TS (Chillo et al., 2008). With increasing of the PANI loaded into the film from $0.02 \mathrm{~g}$ to $0.05 \mathrm{~g}$, the TS decreased initially but increased back at $0.1 \mathrm{~g}$ of PANI loaded into the film irrespective to the size of PANI. PANI is known to be a rigid material when more than $0.02 \mathrm{~g}$ of PANI loaded into the film, the rigid or brittle characteristics of pristine PANI become dominant as the TS of biocomposite film decrease substantially. In addition, this is also due to the insufficient interfacial adhesion material between starch and PANI (Annala and Löfgren, 2006; Nand et al., 2012). Meanwhile, at higher concentration of PANI loaded into the film, $0.1 \mathrm{~g}$, the TS increase. This is due to more molecular mobility between the polysaccharide chains and thus it enhances the initial plastic effect. The free volume between the adjacent chains of polysaccharide is increasing and making the structure more fragile (Maran et al., 2013). In term of the particle size of the PANI, the trends of the TS of the film shows a similar trend for each of the PANI concentration. It is clearly shown that the film containing PANI powder in the range $50 \mu \mathrm{m}$ and $100 \mu \mathrm{m}$ provided higher tensile strength compared to others range size of PANI. The smaller the mean size of particles (as shown in Figure 2(a)) and the narrower the size distribution, the more effectively the particles will enhance the toughness of the matrix. Therefore, small size of the PANI powder is necessary to increase the tensile properties. Unfortunately, the TS of the PANI decrease for the particle size of PANI $100 \mu \mathrm{m} \leq \mathrm{x} \leq 125 \mu \mathrm{m}$ and increase back for the size of PANI $125 \mu \mathrm{m} \leq \mathrm{x} \leq 250 \mu \mathrm{m}$. The increment of TS occurs because the particles containing large particle size (as shown in size distribution in Figure 2(c)) that promote the film damage due to the large pores and crack of the film after stretch.

The EAB of the starch/PANI composites film shows different trends with respective of different concentration for different size of PANI. As a result, the EAB for the particle size of PANI in the range $50 \mu \mathrm{m}$ to $100 \mu \mathrm{m}$ reduces with increasing of PANI loaded. This due to the rigid or brittle nature of small PANI which distribute well through the film hence reduce the flexibility (Bhadra et al., 2017). On the other hand, for sample B1 and $\mathrm{C} 1$ which contained more higher PANI aggregation, showed highest $\mathrm{EAB}$ value. It is due to the movement and rearrangements of the macromolecules chains of the matrix caused the increase in the flexibility (Maran et al., 2013).

\subsection{FTIR spectroscopy}

The FTIR spectra (Figure 3) and their corresponding peak assignments describe the effect of the loading amount and size of PANI on chemical interactions of starch/PANI composites film. The pure starch, starch with plasticizers (glycerol) and PANI powder are also included for the better comparison.

In the pure tapioca starch, the characteristics of the broad band for O-H group (in the presence of H-band) of starch appears at $3252.97 \mathrm{~cm}^{-1}$. Peak around $2924.83 \mathrm{~cm}^{-}$ ${ }^{1}$ is attributed to an asymmetrically starching vibration of $\mathrm{C}-\mathrm{H}$ band in pyranoid ring. It is in line with the FTIR result conducted by Zareh et al. (2011). Several others adsorption bands between $853.88 \mathrm{~cm}^{-1}-1414.67 \mathrm{~cm}^{-1}$ are attributed to the contribution of various functional groups such as $\mathrm{C}-\mathrm{O}$ and $\mathrm{C}-\mathrm{O}-\mathrm{C}$. This result is agreement with the study by Zareh et al. (2011). Meanwhile, the comparison between the pure starch film and starch with additional glycerol showed one different band at a higher wavelength that exhibits the interaction between the starch and glycerol. As seen in Figure 3, there are band changes associated with $-\mathrm{OH}$ groups. This suggested the number increment of oscillation modes that could be attributed to the presence of new hydrogen bonding interactions (Garcia et al., 2009; Liu et al., 2011). The presence of new hydrogen bonding could be interpreted from the peaks $991 \mathrm{~cm}^{-1}$ to $994 \mathrm{~cm}^{-1}$ that shows stronger and narrower band. The double peaks of $\mathrm{O}-\mathrm{C}$ stretching at this band is a result from bending both ' $\mathrm{O}$ ' of $\mathrm{C}-\mathrm{O}-\mathrm{H}$ and ' $\mathrm{O}$ ' of anhydrous glucose ring in starch molecules (Liu et al., 2011).

As depicted in Figure 3, the characteristics peaks of pure PANI were compared in the previous literature (Saikia et al., 2010; Zareh et al., 2011; Bhadra et al., 2017). At $1539 \mathrm{~cm}^{-1}$ and $1456 \mathrm{~cm}^{-1}$ can be assigned to vibration of quinoid ring $(\mathrm{N}=\mathrm{Q}=\mathrm{N})$ and benzenenoid ring (N-B-N), respectively. The bands at $1282 \mathrm{~cm}^{-1}$ and 1230 $\mathrm{cm}^{-1}$ corresponding to $\mathrm{C}-\mathrm{H}$ stretching vibration with aromatic conjugation.

The spectra of starch/PANI composites were very similar to the spectra of pure starch which is in agreement with the study by Nand et al. (2012). Although the presence of PANI in the composites was evident seen with naked eyes and others tests done, peaks that corresponding to PANI were not seen in the spectra of the composites as the PANI loaded was at low 


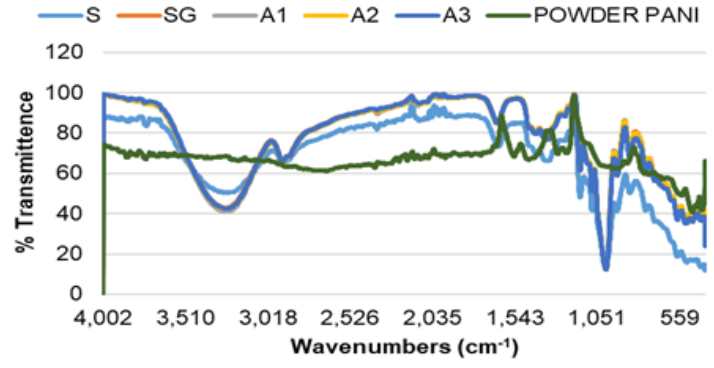

(a)

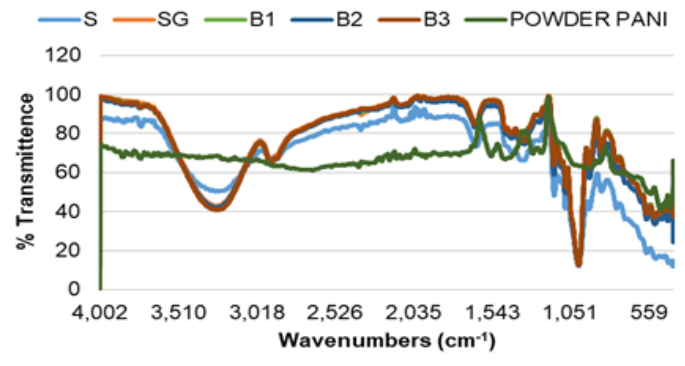

(b)

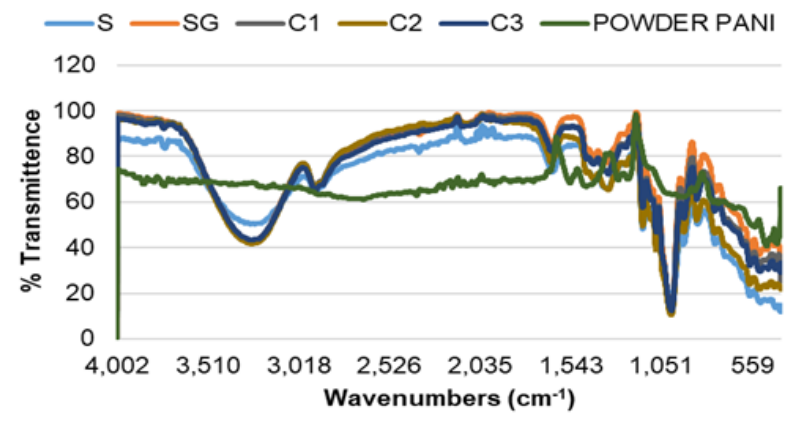

(c)

Figure 3. FTIR spectra of the composites and pristine materials (a) $50 \mu \mathrm{m} \leq \mathrm{x} \leq 100 \mu \mathrm{m}$; (b) $100 \mu \mathrm{m} \leq \mathrm{x} \leq 125 \mu \mathrm{m}$; and (c) 125 $\mu \mathrm{m} \leq \mathrm{x} \leq 250 \mu \mathrm{m}$.

concentration and its distribution would have been largely diluted by the presence of starch. Moreover, it might be due to the solid-liquid condition between the PANI powder and starch that not mixed well like liquidliquid blends of PANI and starch. However, one notable weak pick at $1455 \mathrm{~cm}^{-1}$ which can be found in sample B (Figure 3(b)) and C (Figure 3 (c)) with the respective to the concentration of PANI loaded. This can be due to the contribution from the benzenoid ring (N-B-N) of PANI. In sample A (Figure 3 (a)), this peak was not seen due to the distribution size of PANI was small compared to another sample. The pick near $1149 \mathrm{~cm}^{-1}$ to $1155 \mathrm{~cm}^{-1}$ attributed to the $\mathrm{C}-\mathrm{O}$ stretch in $\mathrm{C}-\mathrm{O}-\mathrm{H}$ prominent in sample $\mathrm{C}$ rather than sample $\mathrm{A}$ and $\mathrm{B}$. This indicates in sample A and B suggests there is loss of moisture absorption capacity of the composites (Saikia et al., 2010).

\section{Conclusion}

In the present work, starch/PANI composite film with different concentration and size of PANI loaded were prepared using solution casting method. As an overall, from the result obtained, concentration and size of PANI loading into the starch/PANI film does give an effect on antioxidant activity, mechanical properties and FTIR result. The biocompatibility of starch/PANI composite film in term of antioxidant is found to be affected by varying the concentration and size of PANI loaded into the film. The composites exhibit an increase in free radical scavenging capacity (a measure of antioxidant activity), with increasing of PANI loading in the film irrespective to the size of PANI. However, the highest free radical scavenging activity occurs at sample B with distribution size of PANI range $100 \mu \mathrm{m} \leq \mathrm{x} \leq 125$ $\mu \mathrm{m}$. By testing the mechanical, free radical scavenging activity and FTIR, it shows that concentration and size of PANI loading do affect the characteristics of the starch/ PANI film. In the mechanical test, the starch/PANI film shows significant difference in term of TS and elasticity properties compared to starch/glycerol film without PANI. Due to the incorporation of rigid and pristine PANI become dominant in the film which affects the strength of the film. Moreover, the TS of the starch/ PANI film becomes higher at higher concentration, $0.1 \mathrm{~g}$ of PANI, due to the molecular mobility between the polysaccharide chains that enhance the initial plastic effect. In contrast with the particle size distributions, the sample shows a decrement at the small size of PANI and increment at bigger size range of PANI loading. This is due to the distribution of the different size of PANI incorporate into the film that gives the effect on the damage and crack on the film. The elasticity of the composites film also shows significant changes with different particles size and concentration. The elasticity of sample B $(100 \mu \mathrm{m} \leq \mathrm{x} \leq 125 \mu \mathrm{m})$ with concentration of $0.05 \mathrm{~g}$ has improved by $21 \%$ compared to the starch/ glycerol film. Based on the FTIR spectra, peak found in the starch/PANI film were similar to the starch/glycerol without PANI are seen due to low concentration and its distribution would have been largely diluted by the presence of starch. However, one notable weak pick at near $1455 \mathrm{~cm}^{-1}$ which can be found in sample B and C with respective to the concentration of PANI loaded. 
This can be due to the contribution from the benzenoid ring (N-B-N) of PANI.

\section{References}

Annala, M. and Löfgren, B. (2006). Compatibilization of conductive polyethylene/ polyaniline blends. Macromolecular Materials and Engineering, 291(7), 848-857. https://doi.org/10.1002/mame.200600071

ASTM. (2002). Standard test method for tensile properties of thin plastic sheeting: Method D882-02. Philadephia: American Society for Testing and Materials.

Bhadra, J., Al-Thani, N.J., Madi, N.K. and Al-Maadeed, M.A. (2017). Effects of aniline concentrations on the electrical and mechanical properties of polyaniline polyvinyl alcohol blends. Arabian Journal of Chemistry, 10(5), 664-672. https://doi.org/10.1016/ j.arabjc.2015.04.017

Cervera, M.F., Karjalainen, M., Airaksinen, S., Rantanen, J., Krogars, K., Heinämäki, J., Colarte, A.I. and Yliruusi, J. (2004). Physical stability and moisture sorption of aqueous chitosan-amylose starch films plasticized with polyols. European Journal of Pharmaceutics and Biopharmaceutics, 58 (1), 69-76. https://doi.org/10.1016/ j.ejpb.2004.03.015

Chang, P.R., Jian, R., Yu, J. and Ma, X. (2010). Fabrication and characterisation of chitosan nanoparticles/plasticised-starch composites. Food Chemistry, 120(3), 736-740. https://doi.org/10.1016/ j.foodchem.2009.11.002

Chillo, S., Flores, S., Mastromatteo, M., Conte, A., Gerschenson, L. and Del Nobile, M.A. (2008). Influence of glycerol and chitosan on tapioca starchbased edible film properties. Journal of Food Engineering, 88(2), 159-168. https:// doi.org/10.1016/j.jfoodeng.2008.02.002

Choi, I., Chang, Y., Shin, S.H., Joo, E., Song, H.J., Eom, H. and Han, J. (2017). Development of biopolymer composite films using a microfluidization technique for carboxymethylcellulose and apple skin particles. International Journal of Molecular Sciences, 18(6), $1278 \mathrm{https} / / /$ doi.org/10.3390/ijms18061278

Garcia, N.L., Ribba, L., Dufresne, A., Aranguren, M.I. and Goyanes, S. (2009). Physico-Mechanical properties of biodegradable starch nanocomposites. Macromolecular Materials and Engineering, 294(3), 169-177. https://doi.org/10.1002/mame.200800271

González, P., Medina, C., Famá, L. and Goyanes, S. (2016). Biodegradable and non-retro gradable ecofilms based on starch - glycerol with citric acid as crosslinking agent. Carbohydrate Polymers, 138, 66
-74. https://doi.org/10.1016/j.carbpol.2015.11.041

Hsu, C.F., Peng, H., Basle, C., Travas-Sejdic, J. and Kilmartin, P.A. (2011). ABTS •+ scavenging activity of polypyrrole, polyaniline and poly(3,4-ethylene dioxythiophene). Polymer International, 60(1), 6977. https://doi.org/10.1002/pi.2912

Hsu, C.F., Peng, H., Zhang, L., Travas-Sejdic, J. and Kilmartin, P.A. (2009). Structural changes in polyaniline upon reaction with DPPH. E-Journal of Surface Science and Nanotechnology, 7, 269-272. https://doi.org/10.1380/ejssnt.2009.269

Hsu, C.F., Zhang, L., Peng, H., Travas-Sejdic, J. and Kilmartin, P.A. (2008). Scavenging of DPPH free radicals by polypyrrole powders of varying levels of overoxidation and/or reduction. Synthetic Metals, 158(21-24), 946-952. https://doi.org/10.1016/ j.synthmet.2008.06.017

Jiménez, A., Fabra, M.J., Talens, P. and Chiralt, A. (2013). Physical properties and antioxidant capacity of starch-sodium caseinate films containing lipids. Journal of Food Engineering, 116(3), 695-702. https://doi.org/10.1016/j.jfoodeng.2013.01.010

Liu, H., Chaudhary, D., Yusa, S.I. and Tadé, M.O. (2011). Glycerol/starch/Na+-montmorillonite nanocomposites: A XRD, FTIR, DSC and1H NMR study. Carbohydrate Polymers, 83(4), 1591-1597. https://doi.org/10.1016/j.carbpol.2010.10.018

Maran, J.P., Sivakumara, V., Sridharb, R. and Immanuel, V.P. (2013). Development of model for mechanical properties of tapioca starch based edible films. Industrial Crops and Products, 42, 159-168. https:// doi.org/10.1016/j.indcrop.2012.05.011

Müller, C.M.O., Yamashita, F. and Laurindo, J.B. (2008). Evaluation of the effects of glycerol and sorbitol concentration and water activity on the water barrier properties of cassava starch films through a solubility approach. Carbohydrate Polymers, 72(1), 82-87. https://doi.org/10.1016/j.carbpol.2007.07.026

Nand, A.V., Ray, S., Easteal, A.J., Waterhouse, G.I.N., Gizdavic-Nikolaidis, M., Cooney, R.P., TravasSejdic, J. and Kilmartin, P.A. (2011). Factors affecting the radical scavenging activity of polyaniline. Synthetic Metals, 161(13-14), 12321237.

https://doi.org/10.1016/ j.synthmet.2011.04.010

Nand, A.V., Ray, S., Travas-Sejdic, J. and Kilmartin, P.A. (2012). Characterization of polyethylene terephthalate/polyaniline blends as potential antioxidant materials. Materials Chemistry and Physics, 134(1), 443-450. https://doi.org/10.1016/ j.matchemphys.2012.03.015

Saikia, J.P., Banerjee, S., Konwar, B.K. and Kumar, A. 
(2010). Biocompatible novel starch/polyaniline composites: Characterization, anti-cytotoxicity and antioxidant activity. Colloids and Surfaces B: Biointerfaces, 81(1), 158-164. https:// doi.org/10.1016/j.colsurfb.2010.07.005

Vieira, M.G.A., Da Silva, M.A., Dos Santos, L.O. and Beppu, M.M. (2011). Natural-based plasticizers and biopolymer films: A review. European Polymer Journal, 47(3), 254-263. https://doi.org/10.1016/ j.eurpolymj.2010.12.011

Wang, J., Zhu, L.H., Li, J. and Tang, H.Q. (2007). Antioxidant activity of polyaniline nanofibers. Chinese Chemical Letters, 18(8), 1005-1008. https:// doi.org/10.1016/j.cclet.2007.05.056

Xu, Y.X., Kim, K.M., Hanna, M.A. and Nag, D. (2005). Chitosan-starch composite film: Preparation and characterization. Industrial Crops and Products, 21 (2), 185-192. https://doi.org/10.1016/ j.indcrop.2004.03.002

Yan, Q., Hou, H., Guo, P. and Dong, H. (2012). Effects of extrusion and glycerol content on properties of oxidized and acetylated corn starch-based films. Carbohydrate Polymers, 87(1), 707-712. https:// doi.org/10.1016/j.carbpol.2011.08.048

Yavuz, A.G., Uygun, A. and Bhethanabotla, V.R. (2009). Substituted polyaniline/chitosan composites: Synthesis and characterization. Carbohydrate Polymers, 75(3), 448-453. https://doi.org/10.1016/ j.carbpol.2008.08.005

Zareh, E.N., Moghadam, P.N., Azariyan, E. and Sharifian, I. (2011). Conductive and Biodegradable polyaniline/starch Blends and Their Composites with Polystrene polyaniline/starch Blends and Their Composites with Polystyrene. Iranian Polymer Journal, 20(4), 319-328. 